\title{
Relaciones académicas y de género en docentes universitarios y su influencia en la publicación en revistas indexadas de alto impacto
}

\author{
Academic and gender relations in university teachers and their influence on \\ publication in high-impact indexed journals
}

Francisco Dillon ${ }^{a}$ ORCID: 0000-0002-8776-3435

Diana Espinosa ${ }^{\mathrm{b}}$ ORCID: 0000-0001-6884-2106

Recibido: 24/9/2019 • Aprobado: 19/11/2019

Cómo citar: Dillon, F., \& Espinosa, D. (2020). Relaciones académicas y de género en docentes universitarios y su influencia en la publicación en revistas indexadas de alto impacto. Ciencia y Educación, 3(3), 7-20. Doi: https://doi.org/10.22206/cyed.2019.v3i3. pp7-20

\section{Resumen}

Las concepciones de género, por lo general, son reproducidas a través de estereotipos y roles que dificultan el trabajo, más que nada a nivel universitario. En el caso de los docentes universitarios, este se ve reflejado particularmente en la escasa participación multidisciplinaria, transdisciplinaria e interdisciplinaria en la autoría y coautoría de artículos indexados en revistas de alto impacto. El objetivo de este estudio fue determinar la influencia de estas relaciones académicas y de género utilizando como marco metodológico la teoría de redes y de género en este tipo de publicaciones. La información utilizada para este análisis, corresponde al número de artículos escrito por docentes investigadores diferenciado por género. Como conclusión se menciona que, las medidas estadísticas de la red de comunicación académica y de género en docentes universitarios, influyen directamente en publicación de este tipo de artículos.

Palabras clave: docente universitario; relaciones académicas; relaciones de género; publicación científica; revista de alto impacto, teoría de redes.

\begin{abstract}
Gender conceptions, in general, are reproduced through stereotypes and roles that hinder work, more than anything at the university level. In the case of university teachers, this is particularly reflected in the low multidisciplinary, transdisciplinary and interdisciplinary participation in the authorship and co-authorship of articles indexed in high impact journals. The objective of this study was to determine the influence of these academic and gender relations using as a methodological framework the theory of networks and gender in this type of publications. The information used for this analysis corresponds to the number of articles written by research professors differentiated by gender. In conclusion, it is mentioned that, the statistical measures of the academic and gender communication network in university teachers, directly influence the publication of this type of articles.
\end{abstract}

Keywords: University teachers; academic relationships; gender relations; scientific publication; high impact magazines, network theory.

\footnotetext{
a Magíster en Educación y Proyectos de Desarrollo con Enfoque de Género. Universidad Tecnológica Indoamérica. Docente de la Maestría en Innovación y Liderazgo Educativo. Quito-Ecuador. Correo-e: franciscodillon@uti.edu.ec

b Economista. Consultora económica y de proyectos educativos y sociales. Asesoramiento Psicológik. Quito-Ecuador.

Correo-e: dianaestefaniaespinosa@gmail.com
} 


\section{Introducción}

\section{Relaciones de género en la colaboración y producción científica}

Las relaciones sociales y académicas de los docentes, por lo general, son definidas por las estructuras de las carreras o programas ofertados en una institución de educación universitaria. En los mismos, se distribuyen horas de docencia, de vinculación con la sociedad y de investigación, asignación de proyectos, o la escritura de artículos que tienden a ser publicados en revistas indexadas (Ponce, 2016). En el Ecuador, principalmente en institutos tecnológicos y universidades, estas relaciones se encuentran estrechamente ligadas a las actividades que cumplen los docentes dentro y fuera del aula de clases, permitiéndoles indagar sobre problemas de la vida real donde se plantean alternativas de solución innovadoras (Buela-Casal \& Sierra, 2007).

Muchas veces, estas relaciones sociales influyen de manera determinante en el accionar académico, $\mathrm{y}$ tienden a reproducir consciente o inconscientemente patrones de género asociados a las estructuras de poder definidas como adecuadas por una cultura paternalista y discriminatoria, especialmente con el género femenino (Dillon, Freire, \& Espinosa, 2019), (Rebollo, García, \& Piedra, 2011). Usualmente, la discriminación de género en el ámbito académico y social tiende a pasar por las siguientes concepciones predefinidas (Dillon \& Espinosa, 2018):

El deber "ser" definido y diferenciado entre hombres y mujeres,

1. la responsabilidad doméstica y de cuidado relegado la mayoría de las veces a las mujeres,

2. los roles de poder ejercidos por varones y la falta de reconocimiento de autoridad y prestigio de las mujeres para la representación y la dirección de cargos importantes,

3. la cultura sexista y de discriminación ejercida en las empresas y organizaciones,

4. la ausencia del enfoque de género en las políticas de empleabilidad y selección de personal en las empresas y organizaciones, $y$;

5. la falta de participación de la mujer en la formación académica y la producción científica.
Por ejemplo, existe evidencia que ha identificado la diferenciación en la producción científica entre varones y mujeres (Cole \& Zuckerman, 1984), (Sonnert, 1995); por un lado, los varones tienden a establecer relaciones académicas menos formales y más perdurables, mientras que, las mujeres, tienden a establecer relaciones académicas menos prestigiosas, lo que les permite de alguna manera ampliar sus conexiones interdisciplinarias y transdisciplinarias (Sonnert, 1995), (Bozeman \& Corley, 2004), ( Fuchs, Stebut, \& Allmendinger, 2001), (Long, 1990), (Leahey, 2006). Este efecto, producto de la discriminación académica diferenciada por género, influye en la autoría y coautoría de artículos científicos en revistas indexadas; conocida como el "Efecto Matilda" (Rossiter, 1993), contraparte del "Efecto Matthew" (Merton, 1968). El efecto Matilda, se manifiesta cuando el reconocimiento resultante de una investigación realizada, pasa a manos de investigadores varones sin considerar el aporte (en igual o mayor grado) de investigadoras mujeres.

Si bien, estas relaciones sociales y académicas se encuentran determinadas por puntos de inflexión comunes como el título profesional, la carga horaria docente diferenciada por carrera, modalidad y sede, las relaciones de amistad y compañerismo establecidos, entre otros; los estudios en los cuales se realizan colaboraciones académicas relacionadas a la publicación científica en revistas indexadas de alto impacto, se encuentran dispersas y limitadas a determinadas áreas de conocimiento (Badar, Hite, \& Badir, 2013), (Long, 1992). Por lo general, estos análisis utilizan metodologías complejas en las cuales recabar información lleva demasiado tiempo y la utilización de variados recursos (Abramo, D’Angelo, \& Caprasecca, 2008), (Boschini \& Sjogren, 2007), (Zippel, 2011), (Shauman \& Xie, 1996).

Las técnicas de análisis de redes sociales permiten caracterizar medidas estadísticas (entre estas, modularidad, los grados de entrada y salida de información, su popularidad), para identificar la propagación de información dentro de la misma (Rubido, Grebogi, \& Baptista, 2017). El análisis de redes sociales (ARS), tiene particular importancia para el entendimiento de sistemas complejos como, por ejemplo, las relaciones sociales y académicas de docentes universitarios, los 
cuales están compuestos por muchos y variados componentes (agentes) que interactúan entre ellos: vinculación, investigación y docencia.

En función de lo mencionado, este estudio pretende analizar las relaciones académicas y de género utilizando como base metodológica el análisis de las relaciones académicas y sociales de docentes universitarios, para correlacionarlas con la producción académica de alto impacto diferenciada por género, esto con el fin de evidenciar, de ser el caso, posibles desigualdades entre hombres y mujeres en el contexto y población tomada como referencia.

\section{Metodología}

El presente trabajo se deriva del proyecto de investigación: "Análisis de la Red Compleja de Interacciones en una Comunidad Universitaria" cuyo objetivo fue estudiar en tres fases la topología de la red de interacciones, de profesores y empleados, dentro del centro de estudios tomado como referencia, y su efecto en la comunicación organizacional. En la primera fase del trabajo se realizó una revisión bibliográfica sobre la teoría de redes y de los métodos involucrados (análisis del estado del arte en la temática, tanto desde un enfoque de la física estadística, de la matemática, de la computación y de la psicología), esto permitió guiar inicialmente la investigación y dar sustento teórico a los modelos y técnicas que fueron empleados. (Aguilar, 2005), (Cestero, 2018). En la siguiente fase, se obtuvo información del sistema que se pretende analizar. La población bajo estudio está compuesta por personal docente y administrativo, quienes constituyen los nodos de la red de interacciones dentro de la comunidad universitaria.

El vínculo o vértice entre los actores se define como la relación que mantienen las personas para desenvolverse en su trabajo en el día a día. Para levantar la información sobre los bordes entre los nodos, se planteó la siguiente pregunta, ¿quién se ha contactado con usted el día de hoy para resolver asuntos del trabajo y cuántas veces lo ha hecho? Ya en la fase de análisis, se utilizó software especializado para el estudio y caracterización de la red obtenida (GEPHI, SOCNET). Estos programas cuentan con licencias gratuitas y abiertas para descarga. Posteriormente, se caracterizó a la red determinando la distancia entre nodos, el coeficiente de agrupamiento, la distribución de grado, y otras magnitudes que permitan dilucidar el comportamiento de la red que contribuye con nuevo conocimiento sobre los procesos en redes reales, en particular respecto de la organización universitaria y su incidencia en la comunicación. (D Blondel, 2008), (Park \& Newman, 2004), (Watts, 2006)

Finalmente, uno de los resultados esperados fue el fortalecimiento de las ciencias básicas y su relación con el estudio de los sistemas complejos. Esto, en el tiempo, podría abrir un espectro amplio de futuros proyectos e incluso, líneas de investigación específicas, en las cuales las áreas de matemática, física, computación, psicología, química y biología trabajen en proyectos de investigación sobre sistemas complejos. (Rubido, Grebogi, \& Baptista, 2017).

Debido a lo mencionado anteriormente, para este trabajo de investigación, se ha definido una metodología de trabajo propio dividida en cuatro fases:

1. Construcción del instrumento (adaptado de la investigación macro anterior) y selección de la muestra;

2. Levantamiento y análisis de información de la topología de la red;

3. Cálculo de las medidas estadísticas que afectan las relaciones sociales, académicas y de género en la producción de artículos de alto impacto publicados en revistas indexadas; $y$,

4. La correlación de las variables intervinientes en las etapas anteriores con la finalidad de establecer marcos comparativos y obtener conclusiones generales del trabajo de investigación.

El enfoque de este trabajo derivado del proyecto de investigación macro, fue de carácter cuantitativo de corte transversal y de alcance:

- Descriptivo: al conocer la red de interacciones y comunicación que influyen en la autoría y coautoría de los artículos académicos en revistas indexadas de alto impacto;

- Exploratorio: al analizar las medidas estadísticas de la red (de interacciones académicas, de género y comunicacionales); $y$,

- Correlacional: al medir la influencia de las medidas estadísticas de la red en la producción de artículos en revistas indexadas de alto impacto. 
Adicionalmente, se tomó como referencia la población docente del anterior estudio (373) y como muestra no probabilística, una selección por conveniencia de aquellos profesores (21) que habían realizado publicaciones indexadas de alto impacto - SCOPUS —, en los últimos 5 años (2014-2019) en los siguientes campos del conocimiento: 1) Administración de Empresas, 2) Biodiversidad y Recursos Genéticos, 3) Computación, 4) Diseño Digital y Multimedia, 5) Ingeniería en Sistemas, 6) Ingeniería Industrial, 7) Educación, y; 8) Psicología (Padua, 2018) para caracterizarlos en la topología de la red (hacerlos visibles).

Se utilizó el instrumento RED_SCOPUS1, adaptado y construido para este estudio, con el cual se aplicaron dos procesos: (a) una prueba piloto cuyo coeficiente de correlación Alfa Cronbach fue de 0.967 (Padua, 2018), (George, 2003), y; (b) la técnica de juicio de expertos. En tal sentido, el instrumento es válido y confiable. Una vez validado el instrumento y aplicada la encuesta, se realizó el análisis de la red de relaciones docentes y de género (Cestero, 2018) y se correlacionaron los resultados de las medidas estadísticas de la topología de la red (grados de entrada y salida, modularidad, page rank, entre otros) con la producción en autoría y coautoría de artículos indexados de alto impacto (SCOPUS) en el periodo de tiempo tomado como referencia en este estudio (D Blondel, 2008) (Aguilar, 2005), (Alda \& Fries, 2005), (Requena, 1998).

Al finalizar esta investigación, los resultados obtenidos, esperan responder a las siguientes preguntas operativas:

- ¿La topología de la red, influye en la comunicación académica y las relaciones de género de docentes universitarios?

- ¿Se evidencian procesos discriminatorios en la escritura de artículos científicos de alto impacto publicados en revistas indexadas donde, la participación de docentes mujeres en la autoría y coautoría no es equitativa?

- ¿Las medidas estadísticas calculadas en la topología de la red, influyen en la autoría y coautoría de artículos científicos de alto impacto publicados en revistas indexadas diferenciados por género?

- ¿La metodología de trabajo utilizada para esta investigación permitió responder a las preguntas de investigación y obtener resultados que evidencian una diferenciación en la autoría y coautoría de artículos científicos de alto impacto publicados en revistas indexadas?

\section{Resultados}

\section{Red de interacciones académicas y de género}

Los actores de una institución de tercer nivel son agentes dinámicos que se asocian entre ellos para producir resultados. Estos, desde el punto de vista sistémico, son fundamentales para articular los ejes sustantivos sobre el cuál la academia se fundamenta. Se puede observar (ver figura 1) la identificación de los 21 nodos docentes que han sido seleccionados y que cumplen con las características determinadas para ser elegidos en este estudio (haber publicado en autoría o coautoría un artículo Scopus en alguna revista indexada en los últimos 5 ańos). Los nodos (puntos de color) representan a las personas que han escrito un promedio de entre 2 a 29 artículos con indexación SCOPUS, mientras mayor es el tamaño del nodo, mayor es el número de artículos publicados (ver figura 1), sin embargo, se deben establecer las medidas estadísticas de la red: grado de entrada y salida, modularidad, page rank; entre otros, para determinar si la comunicación y relaciones de género entre nodos, propicia la producción científica de alto impacto diferenciada como autor y coautor, o por campo amplio y específico de conocimiento (Alda $\&$ Fries , 2005), (Boschini \& Sjogren, 2007), (Shauman \& Xie, 1996), (Abramo, D’Angelo, \& Caprasecca, 2008), (Sonnert, 1995).

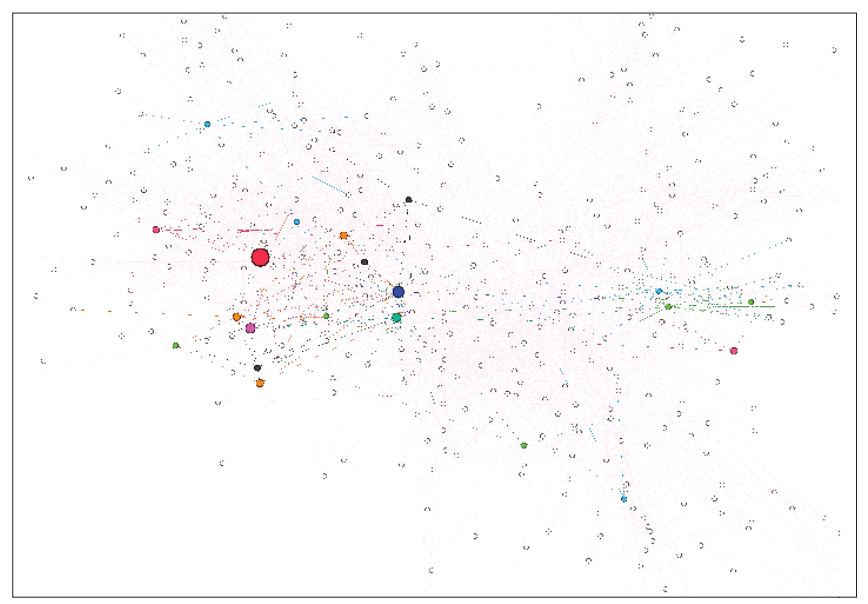




\begin{tabular}{|ll}
0 & $(96,14 \%)$ \\
2 & $(0,92 \%)$ \\
1 & $(0,74 \%)$ \\
3 & $(0,55 \%)$ \\
6 & $(0,55 \%)$ \\
4 & $(0,37 \%)$ \\
9 & $(0,18 \%)$ \\
11 & $(0,18 \%)$ \\
14 & $(0,18 \%)$ \\
28 & $(0,18 \%)$
\end{tabular}

Figura 1. Red de interacciones académicas por número de artículos de alto impacto (SCOPUS) publicado

Fuente: elaboración propia.

Si observamos la caracterización de la topología de la red de docentes diferenciada por género (ver figura 2) el número de docentes varones $(65 \%)$ tiene una mayor cantidad de publicaciones de artículos de alto impacto en revistas indexadas, frente al de mujeres (35\%) (ver figura 3); si a esto le sumamos la diferenciación de este mismo criterio, en función del campo amplio (ver figura 4) y campo específico de conocimiento (ver figura 5), la tendencia respecto a la cantidad de artículos escritos indexados de varones (79 artículos) en relación con las mujeres (30 artículos), se mantiene. Esto, de manera general lo que evidenciaría es una mayor producción científica por parte de docentes varones en comparación con las mujeres; sin embargo, lo importante es recalcar que los procesos comunicacionales (en este caso académicos) inciden de manera directa o indirecta en la producción de artículos publicados en revistas indexadas de alto impacto, ya que mientras mayor sea el número de conexiones de un nodo en la red (enlaces o conexiones de redes académicas), mayor será su probabilidad de relacionarse con más docentes de diversas áreas del conocimiento en la escritura de un artículo indexado de alto impacto, sea como autor o coautor. (Abramo, D’Angelo, \& Caprasecca, 2008), (Betz, 2010), (Badar, Hite, \& Badir, 2013)

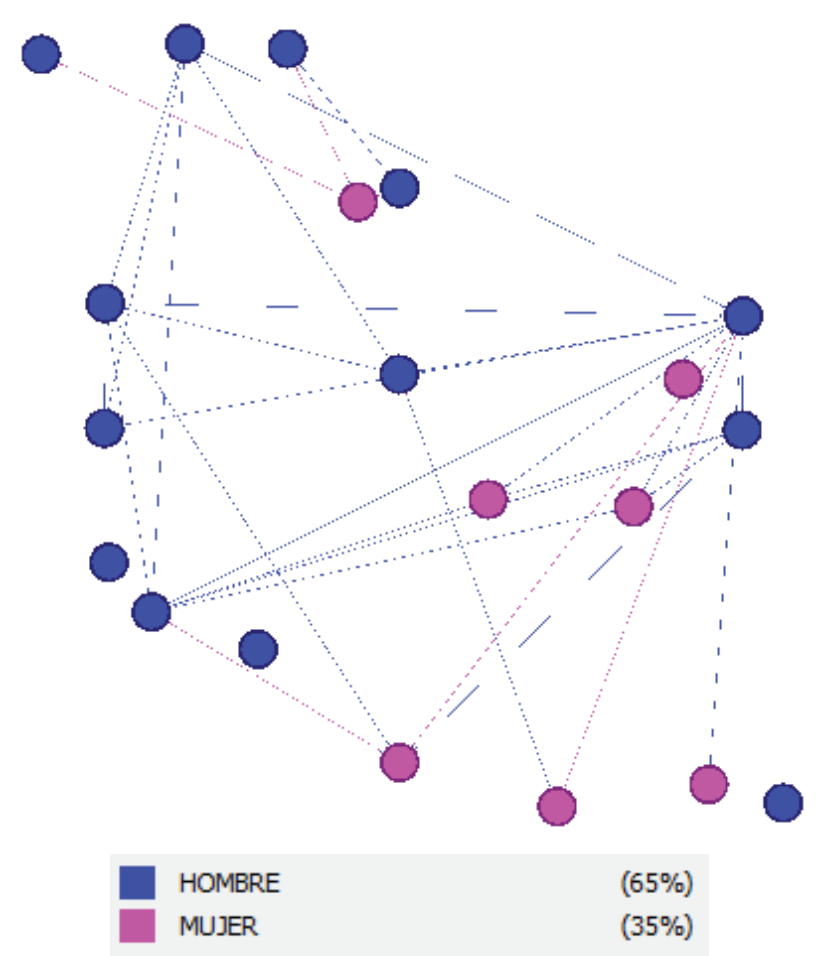

Figura 2. Red de interacciones académicas caracterizada por género, de docentes que mantienen publicaciones de artículos de alto impacto (SCOPUS)

Fuente: elaboración propia.

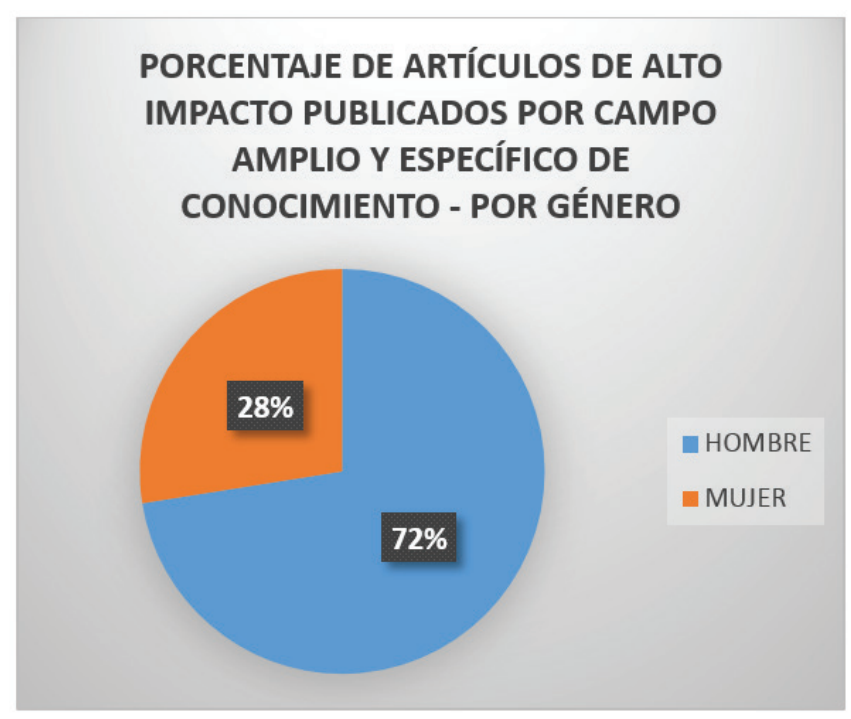

Figura 3. Porcentaje de artículos publicados en revistas indexadas de alto impacto según campo amplio y específico de conocimiento, por género

Fuente: elaboración propia. 


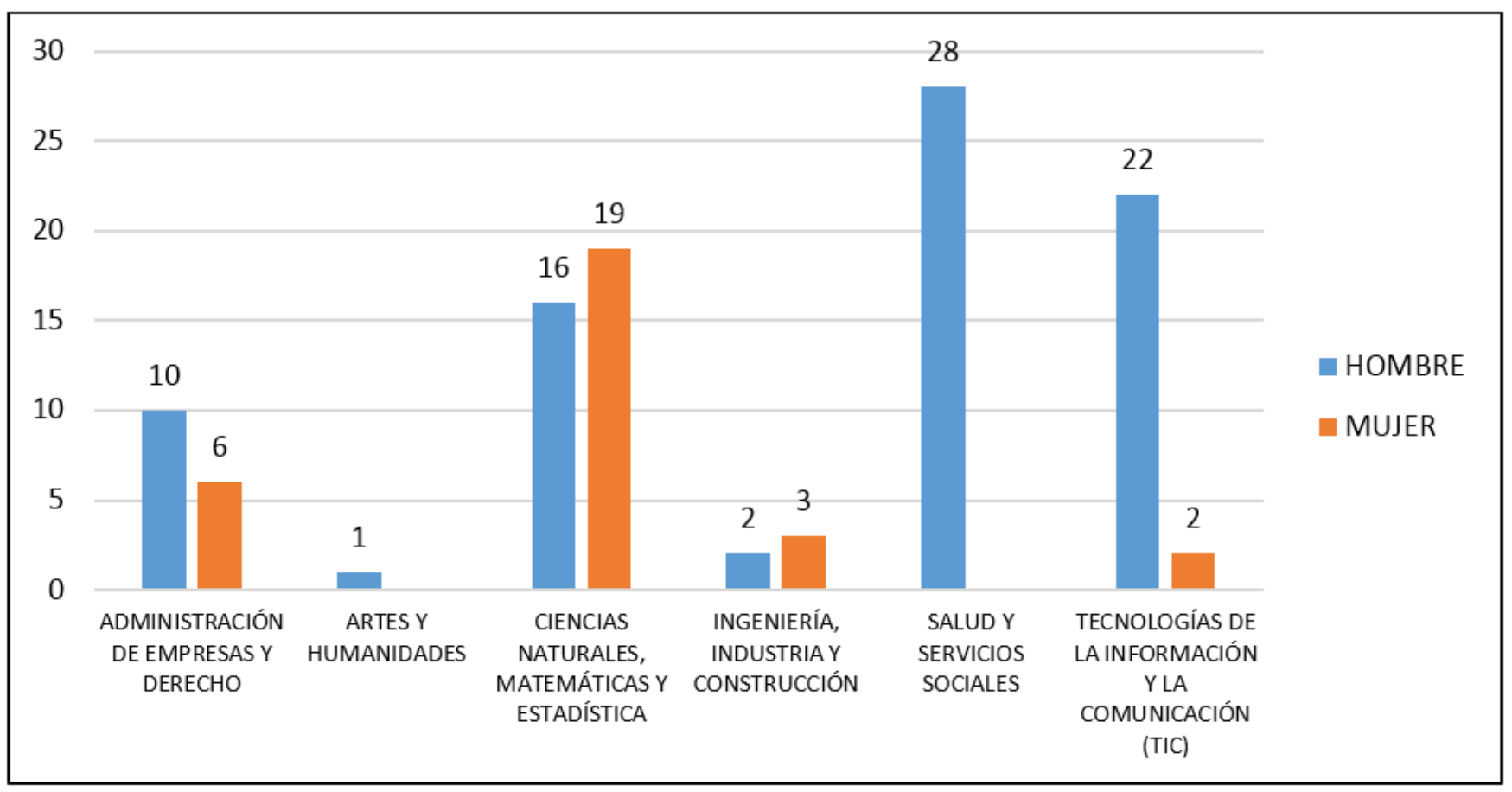

Figura 4. Número de artículos publicados en revistas indexadas de alto impacto según campo amplio de conocimiento, por género Fuente: elaboración propia.

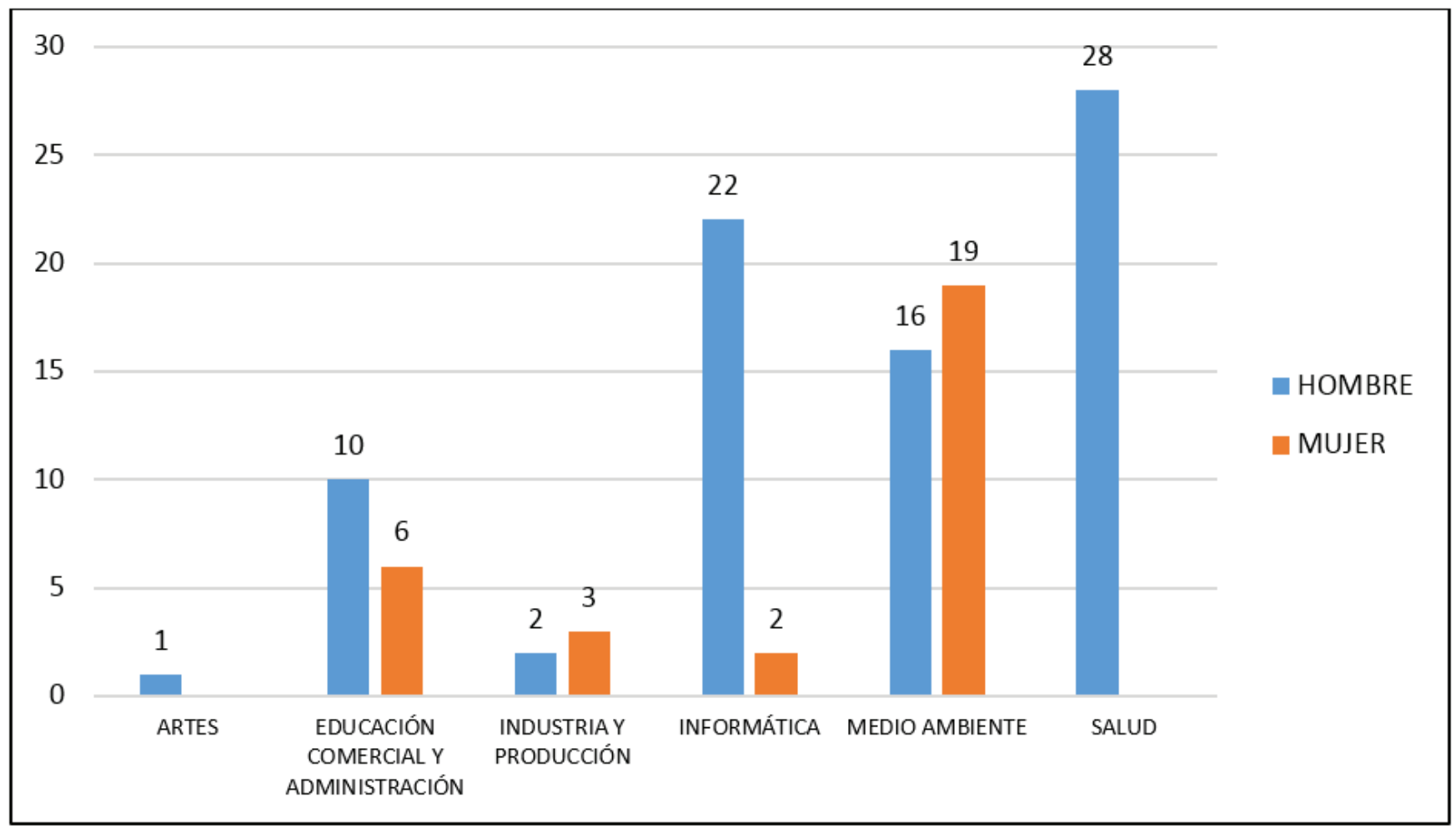

Figura 5. Número de artículos publicados en revistas indexadas de alto impacto según campo específico de conocimiento, por género

Fuente: elaboración propia. 
Con relación a la autoría (ver tabla 1 ) y coautoría (ver tabla 2) de artículos publicados en revistas indexadas de alto impacto, nuevamente se observa una tendencia en la escritura diferenciada por género, por ende, se debe determinar, si el cálculo de las medidas estadísticas en la topología de la red de docentes, incide de manera directa o manifiesta una correlación positiva en la escritura artículos publicados en revistas indexadas de alto impacto diferenciada por género.

Tabla 1. Número de artículos publicados en revistas indexadas de alto impacto según el número de autores diferenciado por género

\begin{tabular}{|c|c|c|c|}
\hline $\begin{array}{c}\mathbf{N}^{\circ} \text { de artícu- } \\
\text { los publicados } \\
\text { como autor }\end{array}$ & $\begin{array}{c}\text { Hombres co- } \\
\text { laboradores }\end{array}$ & $\begin{array}{c}\text { Mujeres colab- } \\
\text { oradoras }\end{array}$ & $\begin{array}{c}\text { Total } \\
\text { general }\end{array}$ \\
\hline 0 & 0 & 0 & 0 \\
\hline 2 & 2 & 0 & 2 \\
\hline 3 & 3 & 0 & 3 \\
\hline 7 & 7 & 0 & 7 \\
\hline Total general & $\mathbf{1 2}$ & $\mathbf{0}$ & $\mathbf{1 2}$ \\
\hline
\end{tabular}

Fuente: elaboración propia.

Tabla 2. Número de artículos publicados en revistas indexadas de alto impacto según el número de coautores diferenciado por género

\begin{tabular}{|c|c|c|c|}
\hline $\begin{array}{c}\mathbf{N}^{\circ} \text { de artículos } \\
\text { publicados } \\
\text { como coautor }\end{array}$ & $\begin{array}{c}\text { Hombres cola- } \\
\text { boradores }\end{array}$ & $\begin{array}{c}\text { Mujeres } \\
\text { colabora- } \\
\text { doras }\end{array}$ & $\begin{array}{c}\text { Total } \\
\text { general }\end{array}$ \\
\hline 1 & 4 & 0 & 4 \\
\hline 2 & 2 & 6 & 8 \\
\hline 3 & 6 & 3 & 9 \\
\hline 4 & 4 & 4 & 8 \\
\hline 6 & 12 & 6 & 18 \\
\hline 7 & 7 & 0 & 7 \\
\hline 11 & 11 & 11 & 22 \\
\hline 21 & 21 & 0 & 21 \\
\hline Total general & $\mathbf{6 7}$ & $\mathbf{3 0}$ & $\mathbf{9 7}$ \\
\hline
\end{tabular}

Fuente: elaboración propia.

\section{Medidas estadísticas estudiadas en las relaciones académicas y de género de la red docente}

Las medidas estadísticas que se analizarán en el presente estudio serán:

\section{Modularidad}

También llamada estructura de comunidad, describe cómo la red se divide en subredes. Se ha demostrado que estas subredes o comunidades tienen un significado dentro del mundo real, es decir, que influyen mucho en una organización o comunidad; (Park \& Newman, 2004). La producción científica acorde a la modularidad, divide en cuatro grupos la red, en la primera tabla (ver figura 6 y tabla 3 ), se observa que los grupos identificados como "0" y “ 3 ” (formados exclusivamente por varones) son los que, en la topología de la red, han sido identificados como los grupos que más producción científica como autores poseen, mientras que, en esta misma medida, la ausencia de las mujeres, es notoria.

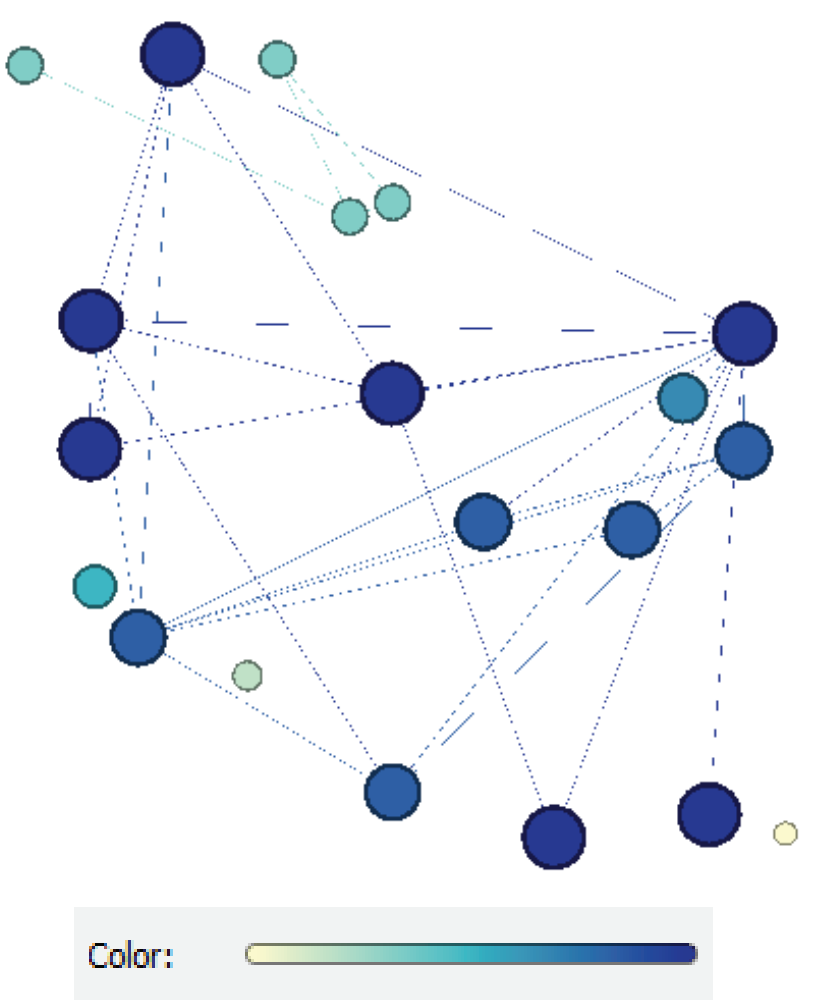

Figura 6. Modularidad de la red docente

Fuente: elaboración propia. 
Tabla 3. Modularidad como autor diferenciado por género

\begin{tabular}{|c|c|c|c|}
\hline \multicolumn{4}{|c|}{ Género } \\
\hline $\begin{array}{c}\text { Grupo definido } \\
\text { por la modularidad }\end{array}$ & Femenino & Masculino & $\begin{array}{c}\text { Total } \\
\text { general }\end{array}$ \\
\hline 0 & 0 & 12 & 12 \\
\hline 1 & 0 & 0 & 0 \\
\hline 2 & 0 & 0 & 0 \\
\hline 3 & 0 & 2 & 2 \\
\hline Total general & $\mathbf{0}$ & $\mathbf{1 4}$ & $\mathbf{1 4}$ \\
\hline
\end{tabular}

Fuente: elaboración propia.

Situación similar sucede en la coautoría de artículos científicos, al parecer, y aunque existe una mayor presencia femenina en la conformación de grupos, se sigue imponiendo la presencia de varones en este tipo de publicaciones (ver tabla 4).

Tabla 4. Modularidad como coautor diferenciado por género

\begin{tabular}{|c|c|c|c|}
\hline \multicolumn{4}{|c|}{ Género } \\
\hline $\begin{array}{c}\text { Grupo definido por } \\
\text { la modularidad }\end{array}$ & Femenino & Masculino & $\begin{array}{c}\text { Total } \\
\text { general }\end{array}$ \\
\hline 0 & 22 & 57 & 79 \\
\hline 1 & 2 & 1 & 3 \\
\hline 2 & 2 & 7 & 9 \\
\hline 3 & 4 & 2 & 6 \\
\hline Total general & $\mathbf{3 0}$ & $\mathbf{6 7}$ & $\mathbf{9 7}$ \\
\hline
\end{tabular}

Fuente: elaboración propia.

La correlación de esta medida estadística dentro de la red, arrojó una correlación significativa $(r=0.56)$ relacionada con la influencia de la conformación de grupos dentro de la red y el grado de salida de los nodos femeninos; esto quiere decir que mientras mayor sea el grado de salida de un nodo femenino en la red, mayor será su probabilidad de formar un grupo académico que escriba indexado de alto impacto, sea como autor o coautor, debido a que los procesos comunicacionales entre redes académicas, son funda- mentales para la escritura de este tipo de artículos (Estébanez, 2007). (Ver figura 7).

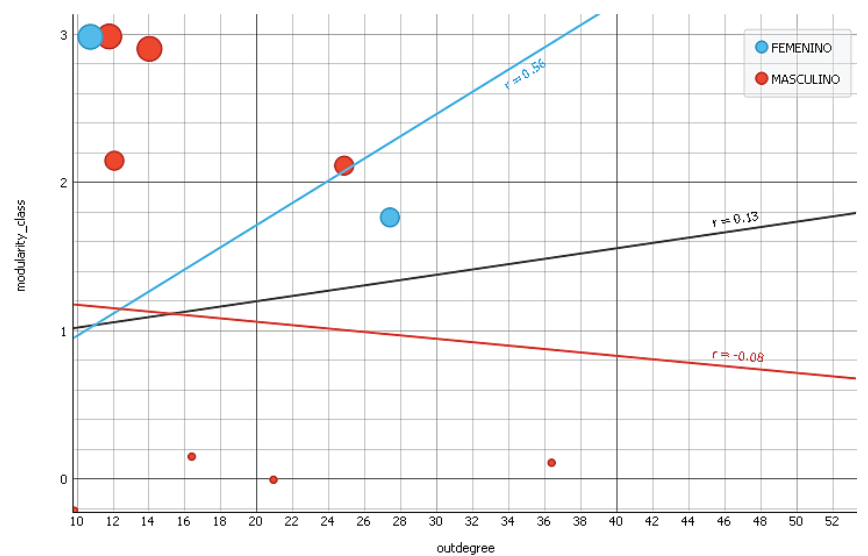

Figura 7. Modularidad y grado de salida

Fuente: elaboración propia.

\section{Page rank}

Es un algoritmo de clasificación para los nodos en un gráfico y permite detectar al nodo que recibe más enlaces y que probablemente sea un nodo importante dentro de la red; (Park \& Newman, 2004), (Rubido, Grebogi, \& Baptista, 2017). La "popularidad de un nodo en la red" de una manera u otra incide en el grado de comunicación (entrada y salida) con el resto de nodos; se puede observar que el page rank más alto se encuentra presente en los varones autores (nodos en la red con color más oscuro), mientras que en la coautoría la distribución es más equitativa entre géneros (ver figura 8 y tabla 5).

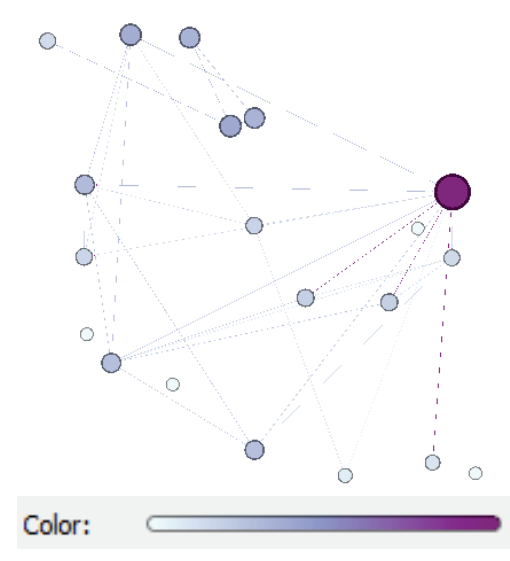

Figura 8. Page rank de la red docente

Fuente: elaboración propia. 
Tabla 5. Page rank diferenciado por género

\begin{tabular}{|c|c|c|c|}
\hline Género & $\begin{array}{c}\text { Total de } \\
\text { publicacio- } \\
\text { nes scopus } \\
\text { - autor }\end{array}$ & $\begin{array}{l}\text { Total de publicaciones } \\
\text { scopus - coautor }\end{array}$ & $\begin{array}{l}\operatorname{Pr}_{-} \\
\text {rank }\end{array}$ \\
\hline Masculino & 0 & 1 & 0 \\
\hline Femenino & 0 & 2 & 14 \\
\hline Masculino & 2 & 0 & 28 \\
\hline Masculino & 3 & 11 & 75 \\
\hline Masculino & 0 & 1 & 0 \\
\hline Masculino & 2 & 7 & 54 \\
\hline Masculino & 0 & 3 & 63 \\
\hline Masculino & 0 & 4 & 2 \\
\hline Masculino & 0 & 2 & 3 \\
\hline Masculino & 0 & 1 & 4 \\
\hline Femenino & 0 & 2 & 3 \\
\hline Femenino & 0 & 3 & 6 \\
\hline Masculino & 0 & 6 & 17 \\
\hline Femenino & 0 & 2 & 29 \\
\hline Masculino & 0 & 1 & 0 \\
\hline Femenino & 0 & 4 & 0 \\
\hline Masculino & 0 & 6 & 29 \\
\hline Femenino & 0 & 11 & 32 \\
\hline Masculino & 7 & 21 & 23 \\
\hline Femenino & 0 & 6 & 23 \\
\hline Masculino & 0 & 3 & 20 \\
\hline
\end{tabular}

Fuente: elaboración propia.

La correlación de esta medida estadística dentro de la red arrojó una correlación altamente significativa en varones $(r=0.98)$ y mujeres $(r=0.83)$. Esta se relaciona a la influencia o popularidad que tiene un nodo dentro de la red y su relación con el grado de entrada; esto quiere decir que mientras mayor sea su popularidad, mayor será su probabilidad de formar un grupo académico que escriba de manera general un artículo, debido a que los procesos comunicacionales entre redes académicas son fundamentales para la escritura de artículos de alto impacto (Estébanez, 2007). (Ver figura 9).

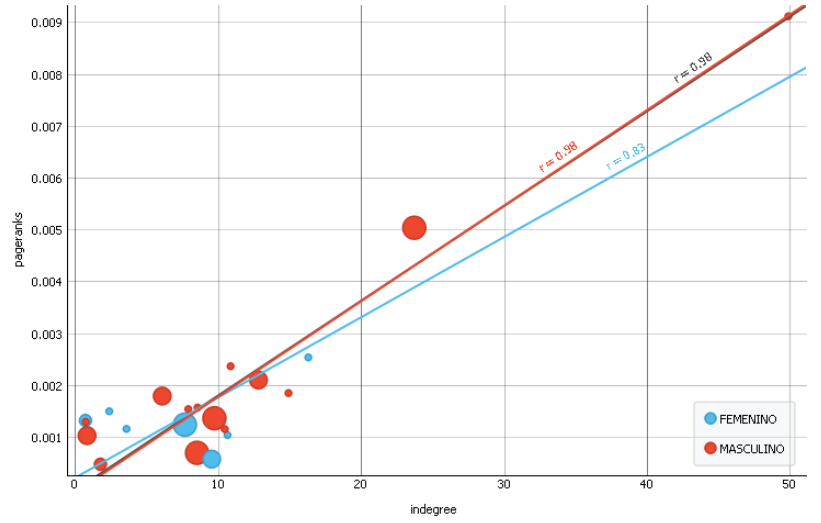

Figura 9. Page rank y grado de entrada

Fuente: elaboración propia.

Grado de centralidad (grado de entrada y salida)

El grado de centralidad permite medir la actividad en la red, es decir, el número de conexiones directas que tiene un nodo y está dividida en grados de entrada (suma de las relaciones referidas hacia un actor por otros) y el grado de salida (es la suma de las relaciones que los actores dicen tener con el resto); (Rubido, Grebogi, \& Baptista, 2017). Se puede observar que dentro de la topología de la red los nodos con color más oscuro son aquellos que mayor grado de entrada y salida manejan, esto quiere decir que en ellos se centran las rutas (canales y medios) por los cuales la comunicación dentro de la red se propaga.

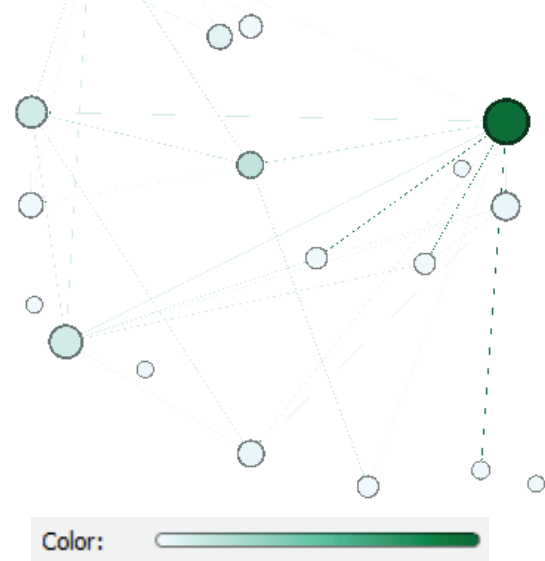

Figura 10. Grados de la red docente

Fuente: elaboración propia. 
Se ha comprobado que la popularidad de un nodo en la red y su grado de entrada y salida facilitan la comunicación entre nodos que propician la escritura de artículos científicos de alto impacto, sin embargo, la conformación de grupos femeninos (ver figura 6) y la cercanía de los nodos en la red (al menos de los nodos masculinos; $r=0.89$ ) (ver figura 11) propician de la misma manera esta escritura y publicación. Sería interesante verificar si esta cercanía se produce debido a la similitud de formación académica, cátedra docente o publicación específica en un área del conocimiento determinado, ya que esto conformaría la premisa de que los docentes se juntan por afinidad académica en la escritura de artículos científicos y no solo por afinidad (Gunnar, 2007).

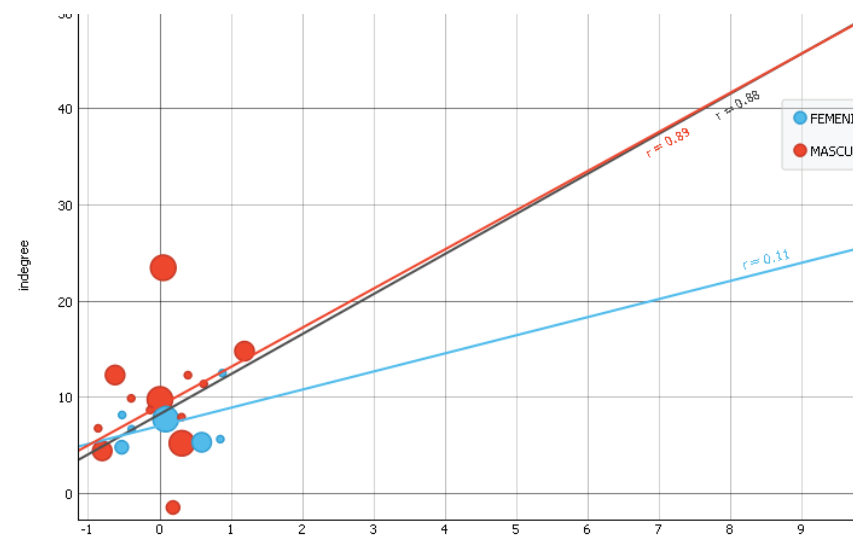

Figura 11. Grado de centralidad y cercanía de los nodos en la red

Fuente: elaboración propia.

\section{Betweenness}

Este grado muestra la periodicidad con que aparece un nodo en el tramo más corto que conecta a otros dos, es decir, muestra la posición intermediaria en las comunicaciones informales entre otras personas del mismo grupo que no se conocen entre sí. Aquellos con mayor intermediación son poseedores de "liderazgo", esto debido a que controlan los flujos de comunicación en la red. (Park \& Newman, 2004) (Rubido, Grebogi, \& Baptista, 2017) (Albert \& Barabási, 2002) En la topología de la red, podemos observar que los nodos con color más oscuro (correspondientes a docentes varones) son los que controlan los flujos de información en la red académica y de género que propicia la escritura de artículos en revistas indexadas de alto impacto, así como por campo amplio y específico de conocimiento dentro de la red (ver figura 12).

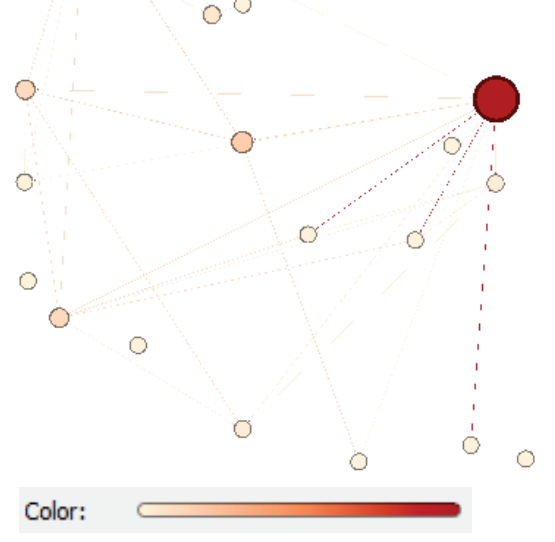

Figura 12. Betweenness de la red docente

Fuente: elaboración propia.

De la misma manera, en esta medida estadística se pudo comprobar que mientras mayor sea el flujo de información que controla un nodo masculino dentro de la red $(\mathrm{r}=0.87)$, mayor será su "popularidad", y, por ende, su posibilidad de conectarse con otros nodos en la red y propiciar comunicación para la escritura de artículos científicos de alto impacto (Estébanez, 2007), (Gunnar, 2007); (ver figura 13).

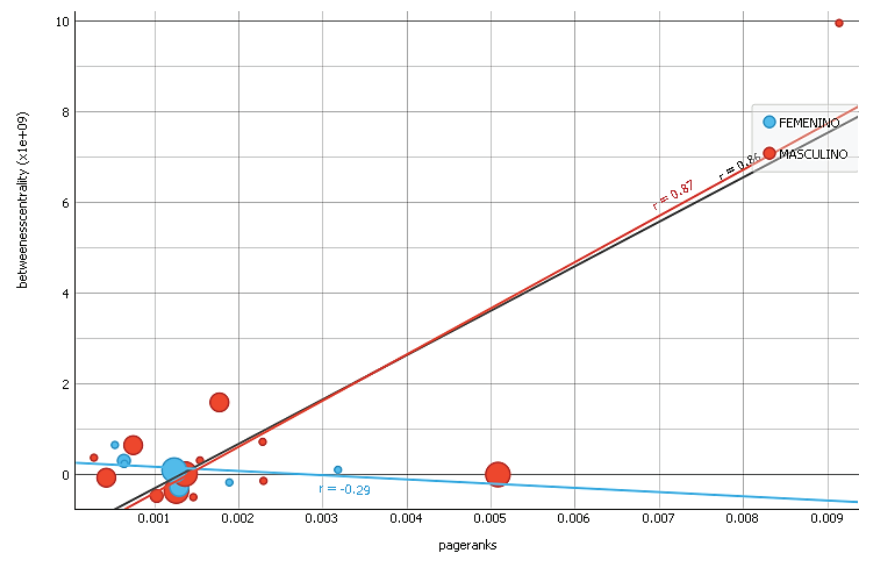

Figura 13. Betweenness y Page rank

Fuente: elaboración propia. 
Eccentricity (Excentricidad)

Son el número de enlaces necesarios para conectar un nodo al nodo más lejano dentro de la red (número de pasos); cuanto mayor es la excentricidad menor es el nodo central (número de pasos que el nodo debe dar para conectarse al nodo más lejano). (Park \& Newman, 2004) (Rubido, Grebogi, \& Baptista, 2017) (Albert \& Barabási, 2002) (Carayol \& Matt, 2004). Se puede observar, en la topología de esta red, que los nodos con color rojo y de menor tamańo, correspondientes casi en su mayoría a docentes mujeres, son las que deben dar el mayor número de pasos para conectarse con otros nodos dentro de la red esta red docente académica (ver figura 14).

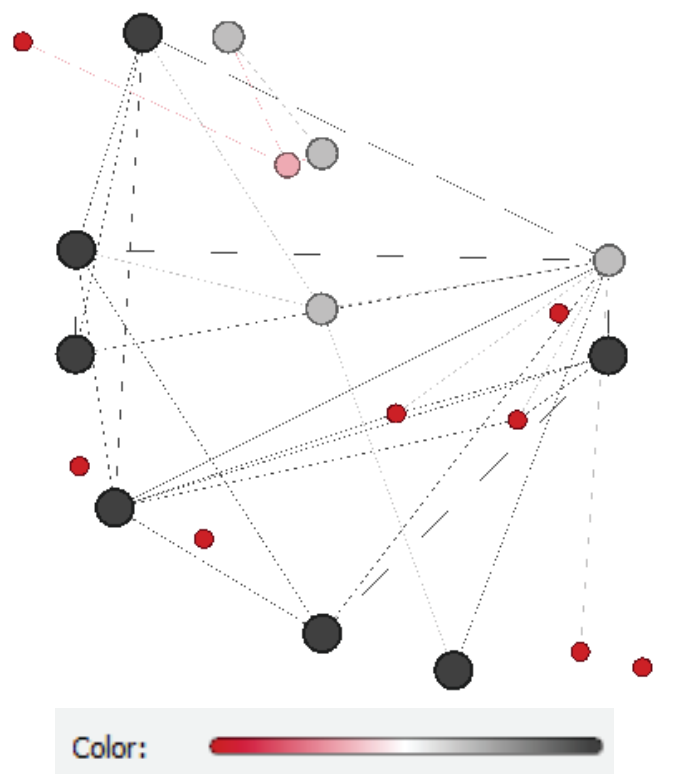

Figura 14. Eccentricity de la red docente

Fuente: elaboración propia.

Además, lo mencionado se puede confirmar dado que en la correlación entre la medida eccentricity y el grado de entrada los nodos femeninos en la red son los menos conectados, esto debido a su grado de entrada y el número de pasos que deben dar para hacerlo $(\mathrm{r}=-0.82)$ (ver figura 15) y que, además, no cuentan con popularidad dentro de la misma $(\mathrm{r}=-0.97)$ (ver figura 16), lo que influye de manera directa al ser los nodos más lejanos y los que más pasos deben dar para comunicarse con el resto de nodos en la red (Carayol \& Matt, 2004), (Estébanez, 2007), (Gunnar, 2007).

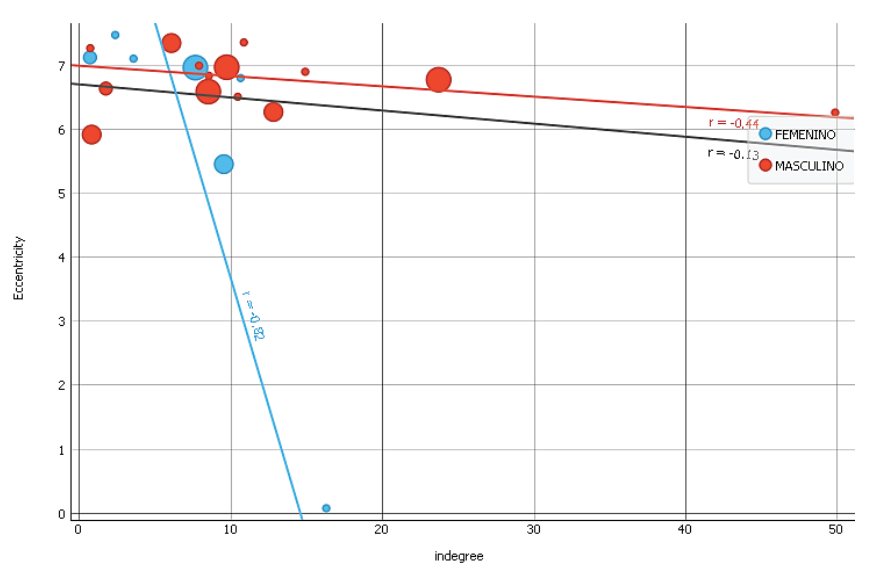

Figura 15. Eccentricity y grado de entrada

Fuente: elaboración propia.

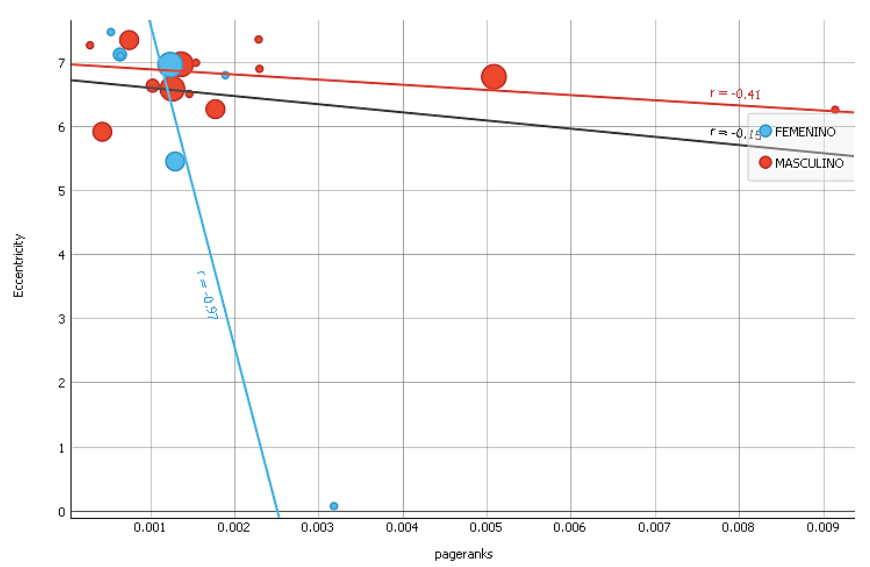

Figura 16. Eccentricity y page rank

Fuente: elaboración propia.

\section{Discusión y Conclusiones}

Las estructuras comunicacionales en las redes académicas que comprenden relaciones de poder entre géneros, definen o determinan de alguna manera la concepción a través de la cual se generan estereotipos que inciden en la publicación y escritura de artículos científicos de alto impacto. Sobre el imaginario social en el que se construyen estas estructuras, subyace aún más profundamente, en el inconsciente, la lucha por el poder, que no permite a hombres y mujeres establecer relaciones sociales y académicas bajo principios de equidad y respeto (Ponce, 2016), (Dillon, Freire, \& Espinosa, 2019) (Rebollo, García, \& Piedra, 2011), 
(Dillon \& Espinosa, 2018), (Alda \& Fries, 2005) (Gunnar, 2007).

Debido a esto, a lo largo del análisis de la topología de la red y sus medidas estadísticas tomadas como referencia en esta investigación, se han encontrado visiblemente sesgos en las estructuras de poder comunicacional de docentes universitarios, diferenciadas por género, esto como resultado final de las relaciones sociales y académicas que propician de manera diferenciada y discriminatoria una mayor escritura de artículos científicos publicados en revistas indexadas de docentes varones. (Abramo, D’Angelo, \& Caprasecca, 2008), (Badar, Hite, \& Badir, 2013), (Boschini \& Sjogren, 2007), (Carayol \& Matt, 2004), (Estébanez, 2007), (Leahey, 2006), (Merton, 1968), (Sonnert, 1995), (Zippel, 2011).

Además, los resultados obtenidos, han permitido responder a las preguntas de investigación planteadas en un postulado anterior:

- ¿La topología de la red, influye en la comunicación académica y las relaciones de género de docentes universitarios? Además, ¿̇se evidencian procesos discriminatorios en la escritura de artículos científicos de alto impacto publicados en revistas indexadas donde la participación de docentes mujeres en la autoría y coautoría no es equitativa?

Sí, esto debido a que se han encontrado diferencias significativas no solamente en el análisis bibliométricos de la producción científica de este tipo de artículos diferenciados por género, sino también en la topología de la red y los flujos comunicacionales que propician la escritura de los mismos como autores y coautores.

- ¿Las medidas estadísticas calculadas en la topología de la red, influyen en la autoría y coautoría de artículos científicos de alto impacto publicados en revistas indexadas diferenciados por género?

Las medidas estadísticas: modularidad, grados de entrada y de salida, page rank, betweenness, y eccentricity, influyen en la topología de la red y los procesos comunicacionales diferenciados por género. Los docentes varones tienen mejores y mayores oportunidades para interre- lacionarse con la red docente que publica artículos de alto impacto en revistas indexadas, y, además, cuentan con una gran popularidad debido a que son los que más publican; en relación con las docentes mujeres, estas no participan de manera directa en la propagación de información de la red académica, deben dar más pasos al encontrarse más alejadas de su propia red y, además, no cuentan con una equitativa participación en la autoría y coautoría de artículos de alto impacto diferenciada incluso, por campo amplio y específico de conocimiento. (Badar, Hite, \& Badir, 2013), (Carayol \& Matt, 2004), (Gunnar, 2007), (Long, Measures of sex differences in scientific productivity, 1992)

- ¿La metodología de trabajo utilizada para esta investigación permitió responder a las preguntas de investigación y obtener resultados que evidencian una diferenciación en la autoría y coautoría de artículos científicos de alto impacto publicados en revistas indexadas? Sí, esto debido a que no solamente se utilizaron medidas estadísticas para determinar los procesos comunicacionales que influyen en las relaciones académicas y de género de docentes universitarios varones y mujeres en la escritura de artículos de alto impacto publicados en revistas indexadas, sino que, además, se analizaron las variables de publicación por autoría, coautoría y campo amplio y específico de conocimiento, lo que permitió evidenciar también desigualdades en los flujos comunicacionales académicos que inciden en la escritura de este tipo de artículos. (Abramo, D’Angelo, \& Caprasecca, 2008), (Rubido, Grebogi, \& Baptista, 2017), (Watts, 2006), (Rossiter, 1993).

Como recomendación, se sugiere que la presente investigación sea trasladada a otros contextos para contrastar los resultados de la red comunicacional diferenciada por género y publicación, no solo de artículos científicos de alto impacto, sino también de libros, capítulos, entre otros; con la finalidad de identificar inequidades de género y cambios en las 
estructuras de poder que no permiten una equitativa participación en la escritura como autores y coautores de artículos multidisciplinarios de alto impacto publicados en revistas indexadas.

\section{Referencias}

Abramo, G., D’Angelo, C., \& Caprasecca, A. (2008). Gender differences in research productivity: A bibliometricanalysis of the Italian academic system. Scientometrics, 79(3), 517-539.

Aguilar, N. (2005). Manual introductorio al análisis de redes sociales. Revista Redes, 29 (1), 16-43.

Albert , R., \& Barabási, A. (2002). Statistical mechanics of complex networks. Reviews of modern physics, 74(1), 47-97.

Alda, F., \& Fries, L. (2005). Feminismo, género y patriarcado. Revista sobre enseñanza del derecho en Buenos Aires, 3(6) 259-294.

Badar, K., Hite, J., \& Badir, Y. (2013). Examining the relationship of co-authorship network centrality and gender on academic research performance: The case of chemistry researchers in Pakistan. Scientometrics, 94(2), 755-775.

Betz, B. (2010). Análisis de las fortalezas institucionales para la contratación de investigadores. Aula Abierta, 38(2), 65-74.

Boschini, A., \& Sjogren, A. (2007). Is team formation gender neutral? Evidence from coauthorship patterns. Journal of Labor Economics, 25(2) 325-365.

Bozeman, B., \& Corley, E. (2004). Scientists'collaboration strategies: Implications for scientific and technical human capital. Research Policy, 33(4), 599-616.

Buela-Casal, G., \& Sierra, J. (2007). Criterios, indicadores y estándares para la acreditación de profesores titulares y catedráticos de universidad. Psicothema 19(4), 537-551.

Carayol, N., \& Matt, M. (2004). Does research organization influence academic production? Research Policy, 33(8), 1081-1102.

Cestero, E. (2018). Data Science y Redes Complejas - Métodos y Aplicaciones. Madrid, España: Editorial Universitaria Ramon Areces.
Cole, J., \& Zuckerman, H. (1984). The productivity puzzle: Persistence and change in patterns of publication of men and women scientists. Advances in motivation and achievement, 1(2), 217-258.

D Blondel, V. (2008). Fast unfolding of communities in large networks. Journal of Statistical Mechanics: Theory and Experiment 1(1), 1-6.

Dillon, F., Freire, I., \& Espinosa, D. (2019). Interacciones de género en la actividad académica de estudiantes de psicología: revelaciones desde la Teoría de sistemas - RED. Wimblu, 14(2), 7-25.

Dillon, F., \& Espinosa, D. (2018). Empleabilidad en el Ecuador desde una perspectiva de género. CienciAmérica, 7(2), 98-112.

Estébanez, M. (2007). Género e investigación científica en las universidades latinoamericanas. Nueva Época, 1(12) 3-26.

Fuchs, S., Stebut, J., \& Allmendinger, J. (2001). Gender, science, and scientific organizations in Germany. Minerva, 39(1) 175-201.

George, D. (2003). Spss for Windows step by step: A Simple Guide and Reference. 11.0 Update. Boston, USA: Allyn \& Bacon.

Gunnar, H. (2007). Gender indicators in science, engineering and technology. An Information Toolkit. Paris, France, UNESCO.

Leahey, E. (2006). Gender differences in productivity. Research specialization as a missing link. Gender \& Society, 20(1), 754-780.

Long, J. (1990). The origins of sex differences in science. Social Forces, 68(4) 1297-1315.

Long, J. (1992). Measures of sex differences in scientific productivity. Social Forces, 71(1), 159-178.

Merton, R. (1968). The Matthew effect in science. Science, 159(3810) 56-63.

Naranjo, A. (2010). Estudio del efecto de la topología de mundo pequeño en el modelo de Ising de 1D y 2D, mediante simulación computacional. Tesis de grado. Quito, Ecuador.

Padua, J. (2018). Técnicas de Investigación aplicadas a las ciencias sociales. Ciudad de México, Mèxico: Fondo de cultura económica. 
Park, J., \& Newman, M. (2004). Statistical mechanics of networks. Physical Review, 70(6), 1-15.

Ponce, J. (2016). Educación Superior en Iberoamérica - Informe 2016. Quito, Ecuador: CINDA - Informe Nacional Ecuador.

Rebollo, M., García, R., \& Piedra, J. (2011). Diagnóstico de la cultura de género en educación: actitudes del profesorado. Revista de Educación, 355(1), 521-546.

Requena, F. (1998). Género, redes de amistad y rendimiento académico. Papers, 56(1), 233-242.

Rossiter, M. (1993). The Matilda effect in science. Social Studies of Science, 23(2), 325-341.

Rubido, N., Grebogi, C., \& Baptista, M. (2017). Understanding Information Transmission in complex networks, 387(1), 1-5.
Shauman, K., \& Xie, Y. (1996). Geographic mobility of scientists: Sex differences and family constraints. Demography, 33(1), 455-468.

Sonnert, G. (1995). Gender differences in science careers. New Brunswick, USA: Rutgers University Press.

Watts, D. (2006). A seis grados de separación. Barcelona, España: Paidós Ibérica, S.A.

Zippel, K. (2011). How gender neutral are state policies on science and international mobility of academics? Sociologica, 1(1), 1-19. 